\title{
Borehole seismic studies of a volcanic succession from the Lopra-1/1 A borehole in the Faroe Islands, northern North Atlantic
}

\author{
Philip Christie, lan Gollifer and David Cowper
}

Extruded basalt flows overlying sedimentary sequences present a challenge to hydrocarbon exploration using reflection seismic techniques. The Lopra-1/1A re-entry well on the Faroese island of Suðuroy allowed us to study the seismic characteristics of a thick sequence of basalt flows from well logs and borehole seismic recordings. Data acquired during the deepening operation in 1996 are presented here.

The re-entry well found that the seismic event at $2340 \mathrm{~m}$, prognosed from the pre-drill Vertical Seismic Profile (VSP) as a decrease in impedance, was not base basalt and the deepened well remained within the lower series basalts. Nonetheless, compressional and shear sonic logs and a density log were recorded over the full open hole interval. These allowed a firm tie to be made with the reflected wavefield from a new VSP. The sonic logs show a compressional to shear wavespeed ratio of 1.84 which is almost constant with depth. Sonic compressional wavespeeds are $3 \%$ higher than seismic velocities, suggesting dispersion in the basalt flows. Azimuthal anisotropy was weakly indicated by the shear sonic log but its orientation is consistent with the directions of mapped master joints in the vicinity of the well.

The VSP downgoing compressional wavelet shows good persistence, retaining a dominant period of $28 \mathrm{~ms}$ at $3510 \mathrm{~m}$ depth. Average vertical velocity is $5248 \mathrm{~m} / \mathrm{s}$, higher than previously reported. Attenuation can largely be modelled by geometrical spreading and scattering loss, consistent with other studies. Within the piled flows, the effective Q from scattering is about 35. Elastic layered medium modelling shows some hope that a mode-converted shear wave may be observed at moderate offsets. Like its predecessor, the 1996 VSP indicates a decrease in impedance below the final depth of the well. However, it is unlikely to be basement or sediment and is probably an event within the volcanic sequence.

Keywords: Faroe Islands, Lopra-1/1A borehole, basalt, vertical seismic profile, seismic attenuation

\footnotetext{
P.C., Schlumberger Cambridge Research, High Cross, Madingley Road, Cambridge CB3 OEl, UK. Formerly: on secondment to BP, Farburn Industrial Estate, Dyce, Aberdeen AB21 7PB, UK. E-mail: pafc1@slb.com

I.G., Fugro-Jason UK Ltd., Unit B Kettock Lodge, Campus 2, Aberdeen Science \& Technology Park, Balgownie Road, Bridge of Don, Aberdeen AB22 8GU, UK. (Formerly: Schlumberger GeoQuest, clo BP, Farburn Industrial Estate, Dyce, Aberdeen $A B 217 P B, U K$.)

D.C., BP Egypt, 14 Road 252, Digla, Ma'adi, Cairo, Egypt. (Formerly: BP, Farburn Industrial Estate, Dyce, Aberdeen $A B 217 P B, U K$.)
} 
The North Atlantic igneous province, of which the Faroe Islands are a part, has been estimated to comprise 10 million $\mathrm{km}^{3}$ of intruded and extruded basaltic igneous rocks (White \& McKenzie 1989). They were emplaced by the processes of rifting and sea-floor spreading which resulted in the opening of this northern part of the Atlantic Ocean. The basalts, which were extruded in a relatively short period of time in the early Palaeogene, cover pre-existing sedimentary rocks which may well be prospective hydrocarbon traps. However, the difficulty of using reflection seismic imaging to probe beneath basalts has been recognised for some time and motivates studies into the characteristics of basalt flows which are relevant for seismic wave propagation. Such studies rely on boreholes which have penetrated significant amounts of basaltic material and in which good quality geophysical logs have been recorded. The Lopra-1/1A research well, on the Faroe Islands, is one such borehole which not only has good quality logs but also has a Vertical Seismic Profile (VSP).

The Lopra-1/1A well-site is located near to the coastline on an isthmus on the southern Faroese island of Suðuroy. The exposed basalt sequence on the Faroe Islands has been divided into a lower, a middle and an upper series, each about $1 \mathrm{~km}$ in thickness (Rasmussen \& Noe-Nygaard 1970, 1990). The $3 \mathrm{~km}$ of exposed lava flows in the Faroe Islands are tholeiitic flood basalts whose extrusion was contemporaneous with the opening of the Norwegian-Greenland Sea in the Palaeogene. The exposed and drilled lower series lava sequence is dated to about $56-59 \mathrm{Ma}$ (Larsen et al. 1999) and is characterised by flows with an average thickness of $20 \mathrm{~m}$, thought to have originated from fissure eruption sites with a NW-SE trend (Rasmussen \& NoeNygaard 1970; Kiørboe \& Petersen 1995). The basalt flows dip to the north-east and the area around the Lopra-1/1A well-site has mapped sets of near-vertical master joints trending NW-SE and NE-SW. The Lopra-1/1A location was selected for probing the deep basalt layers and their substrata because the ground surface is about $750 \mathrm{~m}$ below the top of the lower series.

The Lopra-1 research borehole was originally drilled to a total depth (TD) of $2178 \mathrm{~m}$ in 1981. It was logged and suspended with most of the drilled interval left uncased. The well was surveyed by the Geological Survey of Denmark with a zero-offset VSP and a walkaway VSP, acquired by Prakla-Seismos, in 1988. A refraction profile was acquired by the Faroe Islands Natural History Museum and the University of Bergen in 1989. The results and interpretation are summarised by Kiørboe \& Petersen (1995). In 1996, the well was re-entered and deepened in a number of stages by the Lopra Deepening Consortium. The original well was extended to $3158 \mathrm{~m} \mathrm{~KB}$ (measured depth rela- tive to the Kelly Bushing) using a larger rig with KB 16.2 $m$ above mean sea level, retaining the original name Lopra1. For technical reasons, a side-track, Lopra-1A, was drilled from $3091 \mathrm{~m} \mathrm{~KB}$ to TD at $3565 \mathrm{~m} \mathrm{~KB}$. The well was logged several times during drilling, and logs were run in both the side-track and the original well. In this paper, we deal with data from a composite of the log runs in both the 1 and $1 \mathrm{~A}$ wells, and therefore we use the name Lopra-1/1A re-entrywell or the Lopra-1/1Adeepening for the combined extensions drilled in 1996.

One goal of the Lopra project was the seismic characterisation of piled basalt flows. In the event that significant siliciclastic sediments were encountered beneath the basalt sequence, the consortium partners had agreed on a programme of multiple-azimuth, walkaway VSPs to characterise the properties of compressional and shear wave transmission and reflection at a basalt-sediment contact as functions of both the vertical polar angle and the horizontal azimuthal angle. Layered systems of high velocity contrasts, such as basalt flows, are expected to exhibit transverse isotropy with an axis of symmetry perpendicular to the layering, at wavelengths long compared to the layer thickness. Such anisotropy has its fastest velocity in the direction parallel to thelayering. Kiørboe \& Petersen (1995) had reported velocities higher in the vertical direction than the horizontal and offered an explanation in vertical fractures around basalt columns, possibly in combination with the nearly vertical master joints. Such vertical fractures, if aligned, would be expected to result in an azimuthal variation of seismic velocity which should be fastest in the direction parallel to the fractures.

In fact, neither sediment nor basement was encountered in the well and so the borehole seismic programme was confined to a short-offset VSP and check shot survey designed to measure the short offset reflectivity at the well and to identify depths of intermediate reflectors penetrated by the bit. In particular, Kiørboe \& Petersen (1995) had reported a reflection on the VSP at an interpreted depth of $2340 \mathrm{~m}$ which was thought to result from a decrease in impedance. Such a reversal in impedance might have corresponded to the base of basalt/top of sediment but turned out not to be the case. Further objectives of the borehole seismic analysis were to calibrate the sonic log, thereby providing a detailed velocity-depth model, and to estimate the seismic attenuation of the basalt sequence.

The VSP was complemented by the acquisition over the full interval of the well of compressional $(\mathrm{P})$ and shear (S) wave sonic logs, acquired in four component mode to estimate azimuthal anisotropy parameters (Esmersoy et al. 1994), and a density log. The log data enabled a good well-tie to be made and allowed a modelling study to sup- 
port estimates of anisotropy parameters and attenuation.

This paper presents a summary of the data acquisition, compares the results with those reported in previous studies and discusses their interpretation in the context of the seismic characteristics of piled basalt flows. It draws upon previously unpublished data and reports analysed and compiled by the authors for BP, their Atlantic Margin partner Shell, and their associates in the Lopra Deepening Consortium.

\section{Data acquisition}

The earlier VSP surveys (Kiørboe \& Petersen 1995) had found evidence for a strong seismic reflector at $2340 \mathrm{~m}$, just below the $1981 \mathrm{TD}$, interpreted as a reversal in impedance. This event, and other deeper events seen on the VSPs, were targets for the deepening of the well in 1996. Further motivation was given by the discovery of methane and nitrogen at a pressure of 20 bar when the well was re-opened in 1983 (Kiørboe \& Petersen 1995). As mentioned above, the contingency plan for the 1996 deepening project called for offset VSPs at multiple azimuths in the event of finding $200 \mathrm{~m}$ or more of siliciclastic sediments. Exploring a range of offsets was intended to evaluate the angular dependence of $\mathrm{P}$ - to $\mathrm{S}$-mode conversions in transmission within the basalt sequence, and their possible conversion back to $P$ at the top of any basalt-sediment contact encountered. This would test the applicability to the Faroese basalts of PS-mode converted imaging, as later reported by Emsley et al. (1998).

Kiørboe \& Petersen (1995) had reported that the vertical P-velocity was about $10 \%$ faster than the horizontal P-velocity in the upper $800 \mathrm{~m}$ of the basalt sequence and appealed to fractures to explain this difference. It was hoped that the new VSP would be able to explore the nature of the vertical and azimuthal anisotropy throughout the interval of the deepened well. The location of the Lopra-1/1A well on an isthmus would have facilitated the use of a marine mobile source but because of the establishment of a fish hatchery in the fjord, the 1996 VSP could not make use of a marine airgun and so twin Vibroseis units were shipped to the Faroe Islands for land walkaway VSPs in different azimuths.

In the event, Lopra-1A TD remained in the lower basalt series and so a short-offset VSP was acquired by Schlumberger on 29 October 1996 from 3510 m KB to 1320 m $\mathrm{KB}$ with additional checkshots up to $200 \mathrm{~m}$, using the two Vibroseis sources in tandem. The Vibroseis units swept from $10 \mathrm{~Hz}$ to $130 \mathrm{~Hz}$ over 16 seconds and 2-4 sweeps were recorded at each level using a sample interval of $2 \mathrm{~ms}$.
In addition, checkshots were recorded using an airgun source in a water filled pit to calibrate the Vibroseis transit times. The downhole tool used gimballed triaxial geophones mounted in a sensor package decoupled from the body of the tool. The deepened well was surveyed at $20 \mathrm{~m}$ intervals for VSP waveform processing. The cased hole section was surveyed at similar intervals to a point above the cement top where it became clear that the casing was unsupported and no longer well coupled to the formation.

Some logs recorded after drilling the original well were uncertain in their calibration. The sonic log was a cement bond tool with a single source-receiver pair and lacked shear sonic information (Nielsen et al. 1984). Since a goal for Lopra-1/1A was to characterise seismic propagation characteristics for basalt flows, a set of new logs were acquired over the original open hole section, prior to setting 7-inch casing and drilling on with a 6.5-inch bit. All the drilled intervals were logged with density, $\mathrm{P}$ - and S-sonic from a dipole shear tool in four component mode allowing estimates of azimuthal anisotropy, and a formation microscanner. The logs are not subject to petrophysical interpretation in this paper, but were used for geophysical analysis.

\section{Results}

\section{VSP}

The raw VSP traces were correlated, edited and vertically summed to produce a stacked trace at each level. The stacked vertical geophone data are displayed in Fig. 1a in one-way time, static corrected to mean sea level. From the top of the VSP down to approximately $1840 \mathrm{~m} \mathrm{~KB}$, the waveforms following the first arriving compressional wave are affected by borehole reverberations caused by unsupported casing in a hard rock environment with a non-attenuative fluid in the hole. The amplitude data in this interval are treated with caution, though the arrival times appear to be representative of basalt velocity.

Below the cement top, the stacks show good waveform consistency from level to level. Clearly visible are:

A. down-going multiples (parallel to, but later than, the first arrivals);

B. up-going primary reflections, both within and below the drilled interval. These are characterised by an almost linear moveout of equal slope but opposite sign to that of the first arrivals;

C. suggestions of down-going shear energy, with a linear moveout greater than that of the first arrivals;

D. a weak tube-wave (visible only above the cement top). 

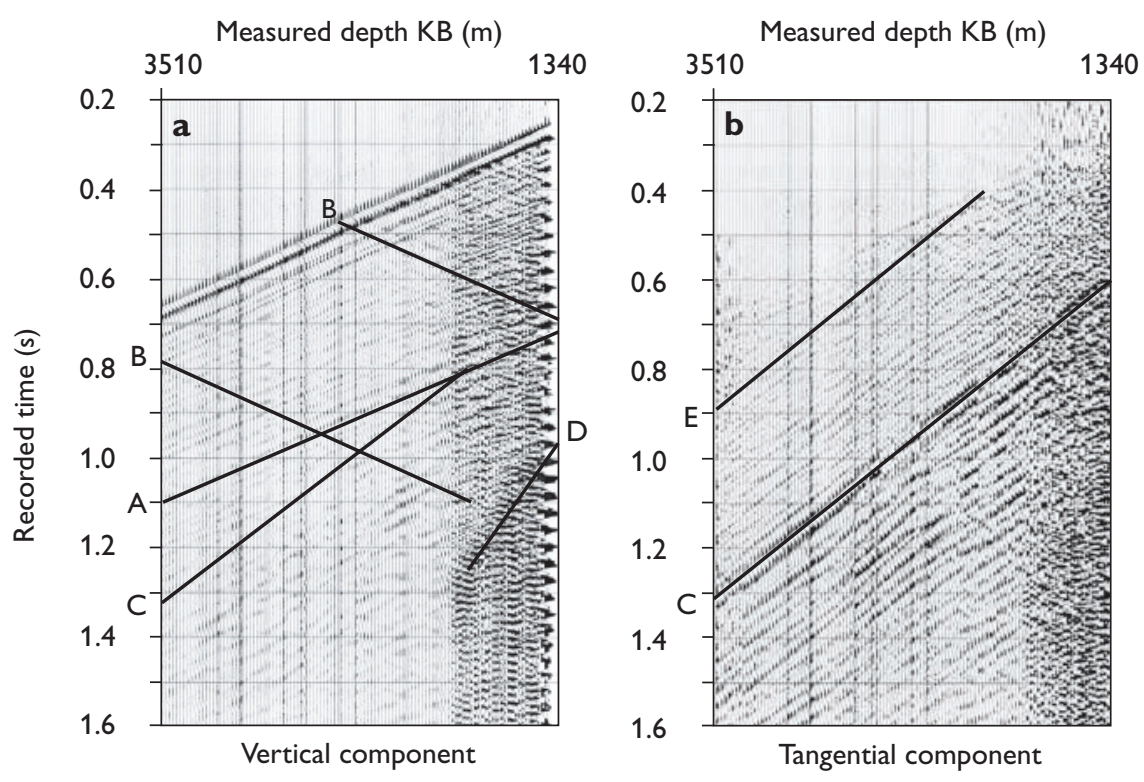

Fig. 1. a: Lopra-1/1A VSP: stacked vertical component geophone data. b: Lopra-1/1A VSP: stacked tangential component geophone data. Labelled phases are described in the text.

The vertical one-way time from mean sea level to the deepest VSP level at $3510 \mathrm{~m} \mathrm{~KB}$ (equivalent to $3478 \mathrm{~m}$ True Vertical Depth Sub-Sea: TVDSS) is $663 \mathrm{~ms}$, corresponding to a compressional velocity of $5248 \mathrm{~m} / \mathrm{s}$ over the interval. This is higher than values for basalt velocity reported elsewhere in the literature (e.g. Planke \& Cambray 1998 ) and higher than the $4.35 \mathrm{~km} / \mathrm{s}$ average velocity estimated from the first logs run in Lopra-1 (Nielsen et al. 1984). This is partly due to the high velocity dolerites and partly due to the relatively thick flows in Lopra$1 / 1 \mathrm{~A}$, giving a higher thickness ratio of fast, flow-centre material to slower, flow-boundary material. As discussed later, the average compressional wavespeed from the sonic log is slightly higher, supporting the VSP observation.

The trace scaling in Fig. 1a is constant for all levels, revealing the total amplitude loss with depth in the first arrivals. Although the VSP interval, from $3510 \mathrm{~m} \mathrm{~KB}$ to $1320 \mathrm{~m} \mathrm{~KB}$, comprises $2174.5 \mathrm{~m}$ TVD of stacked basalt flows, the amplitude loss from geometrical spreading, scattering and attenuation still leaves a good level of signal above the noise floor in the deepest section.

Fig. $1 \mathrm{~b}$ shows the $\mathrm{Y}$-component stacks ( $\mathrm{Y}$ is the horizontal component tangential to the borehole wall) on which the shear energy is most evident. The strongest arrivals are down-going direct shear arrivals, generated by the vertically polarised vibrator acting on the rigid surface. The average shear velocity across the logged interval is about $2900 \mathrm{~m} / \mathrm{s}$, resulting in an average $\mathrm{V}_{\mathrm{P}} / \mathrm{V}_{\mathrm{S}}$ ratio of 1.8 , which is in good agreement with the value of $1.84 \pm$ 0.01 (one standard deviation) estimated below from the sonic $\log$ regression of $V_{S}$ upon $V_{P}$.

Also visible on the Y-component stacks are down-go- ing mode-converted shear events, E, generated by impedance contrasts crossed by the drill-bit. These events are parallel to and earlier than the direct shear arrival, C, but originate at the time and depth of the direct compressional wave's encounter with the mode-converting impedance contrast. These arrivals have a frequency content similar to the associated $\mathrm{P}$-wave and higher than the frequency of the direct shear wave due to the lower cumulative attenuation.

Finally, some weak up-going reflected shear events can be seen. No further processing of the shear arrivals has been performed.

The VSP P-wave data were processed through a workflow comprising trace editing, vertical stacking at each depth level, correction to mean sea level, spherical divergence correction, up/down wavefield separation and deconvolution to zero-phase wavelets with bandwidths of $10-70 \mathrm{~Hz}$ and $10-40 \mathrm{~Hz}$. The former maximises the resolution at the cost of more high frequency noise, while the latter minimises residual noise at the expense of bandwidth. Correlations of the $10-70 \mathrm{~Hz}$ up-going wavefield are presented and discussed in the well-tie section.

\section{Geophysical log data}

In this section we examine the $1996 \log$ data and their correlations to infer the seismic properties of the basalt and to compare with the properties of similar basalts penetrated in Hole 917A of the Ocean Drilling Programme, as reported by Planke \& Cambray (1998).

The P- and S-sonic and density logs, spliced from the 
Fig. 2. P-velocity, S-velocity and density logs, upscaled to $3 \mathrm{~m}$ sample interval using a Backus average (Backus 1962) and displayed in measured depth relative to KB. Core points are plotted from data supplied by GEUS for comparison.

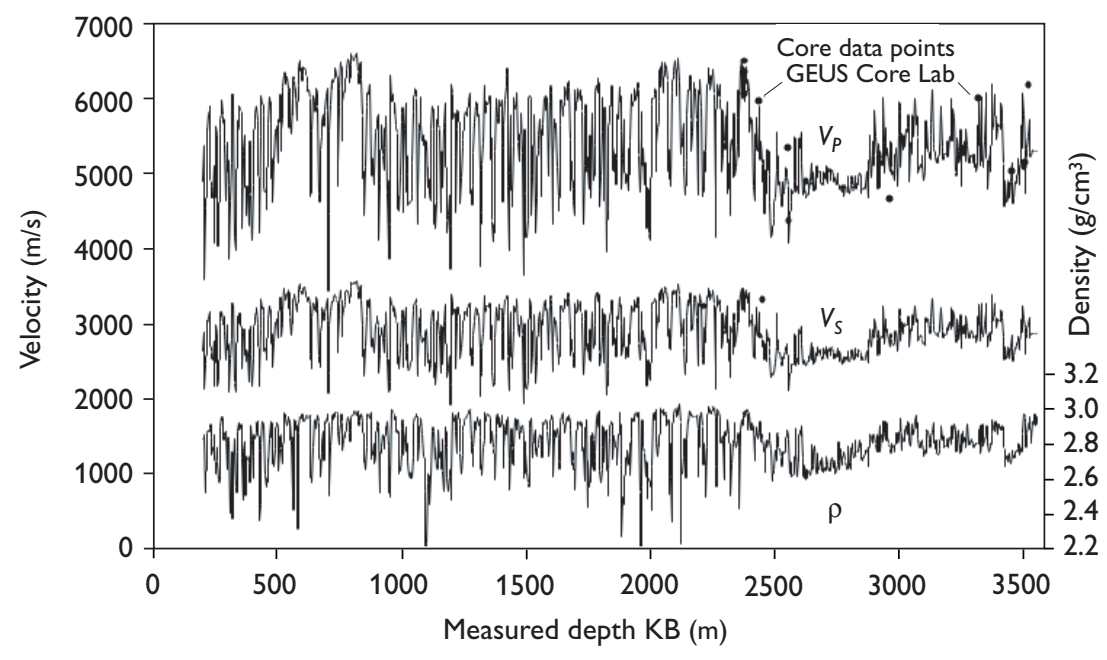

several logging runs made in the 1 and $1 \mathrm{~A}$ wells, are shown in Fig. 2 on a measured depth scale relative to KB. The well is close to vertical, so no true vertical depth corrections have been made for this part of the analysis. The logged section comprises $3371 \mathrm{~m}$, with the dipole shear tool logged in four-component mode together with the density tool. The logs are of good quality and allow the identification of many subaerially emplaced flows over much of the section. Figure 2 shows the P-and S-velocity and density logs after re-sampling the data to $3 \mathrm{~m}$ using a Backus average (Backus 1962; Folstad \& Schoenberg 1992). There are wide variations in $\mathrm{P}$ - and S-velocities, in an asymmetric, quasi-periodic manner, though the mean velocities are very consistent over the well. The boundaries of each flow are characterised by a shift to lower velocities and density, caused by the formation of vesicles at the top and base of the flow (Planke \& Cambray 1998), and possibly in places by weathering, alteration and rubble. The main exceptions to this character are the high velocity dolerite intrusions, encountered at about $600 \mathrm{~m} \mathrm{~KB}$ and $770 \mathrm{~m}$ $\mathrm{KB}$, and the zone of almost constant velocity and density between about $2600 \mathrm{~m} \mathrm{~KB}$ and $2900 \mathrm{~m} \mathrm{~KB}$, corresponding to a thick, hyaloclastite sequence.

The dots near the compressional velocity log ( $\mathrm{Vp}$ in Fig. 2) mark depths and values of ultrasonic measurements of P-wave velocity made by GEUS on a number of core samples. The measurements were taken on $25 \mathrm{~mm}$ core samples, pressure-saturated with distilled water and using piezo-electric transducers of centre frequency $1 \mathrm{MHz}$ (2380 m core) and $2.5 \mathrm{MHz}$ (all other cores) at room temperature and pressure. Although the scale of the display makes a visual match difficult, it can be seen that there is generally a good correlation between the core measurements of P-wavespeed and the log data. With only two or three exceptions, at $2218 \mathrm{~m} \mathrm{~KB}, 2455 \mathrm{~m} \mathrm{~KB}$ and $2972 \mathrm{~m}$
$\mathrm{KB}$, the core measurements are either the same as the log values or a little faster, consistent with dispersion of the higher frequency core measurement compared to the sonic log.

\section{Discussion}

\section{Layer-induced anisotropy}

Backus upscaling applied to a stack of isotropic layers predicts a transversely isotropic model having a symmetry axis perpendicular to the layers. The upscaled elastic parameters can be recast in terms of Thomsen's (1986) weak anisotropic parameters. Figure 3 shows the modelled values of epsilon $(\varepsilon)$, and delta $(\delta)$, after upscaling the logs to $3 \mathrm{~m}$. The parameters are given by the relations:

$$
\varepsilon=\frac{C_{11}-C_{33}}{2 C_{33}}
$$

and

$$
\delta=\frac{\left(C_{13}+C_{33}\right)\left(C_{13}-C_{33}+2 C_{55}\right)}{2 C_{33}\left(C_{33}-C_{55}\right)}
$$

where

$$
C_{33}=p V_{p}^{2}, \quad C_{55}=p V_{s}^{2}, \quad C_{13}=C_{33}-2 C_{55}
$$

and $\rho, V_{P}$ and $V_{S}$ are the Backus-upscaled log values of density, compressional wavespeed and shear wavespeed. Epsilon is the measure of axial compressional anisotropy, while delta controls the off-axis behaviour of the phase velocity near to the vertical. Both enter the following relation from Thomsen (1986) governing the compressional phase velocity behaviour as a function of angle $\theta$ to the (vertical) axis of symmetry: 


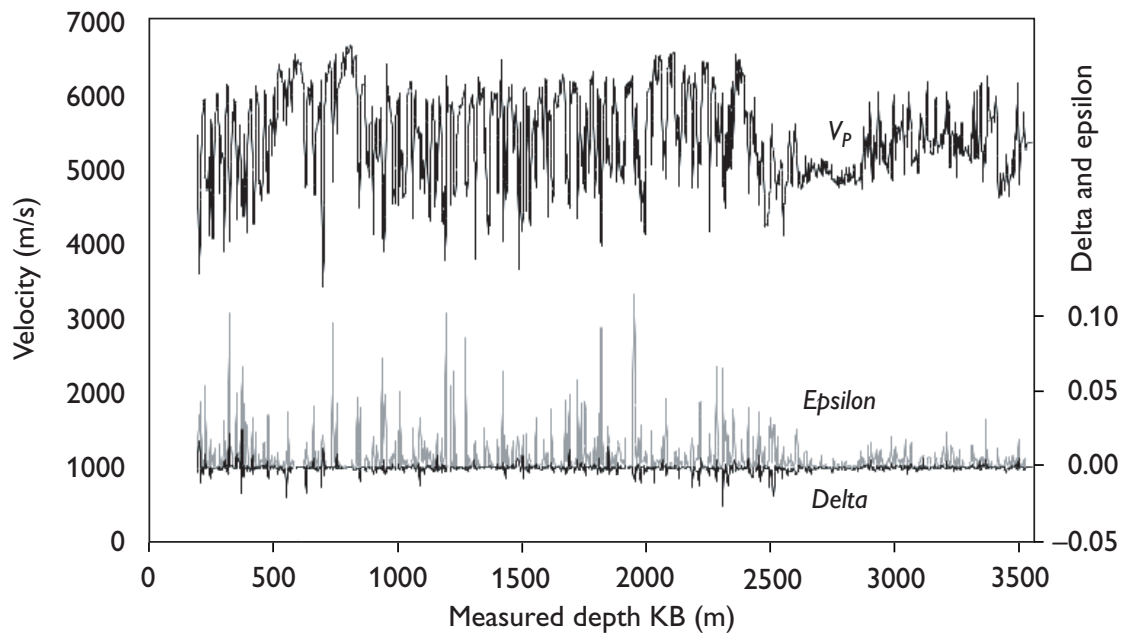

Fig. 3. Log of Thomsen's (1986) weak anisotropic parameters epsilon and delta, modelled from the Backus upscaling of the $15 \mathrm{~cm}$ logs to $3 \mathrm{~m}$, assuming isotropic individual layers. The compressional sonic velocity is shown for correlation, also upscaled to $3 \mathrm{~m}$.

$$
V_{P}(\theta) \approx V_{P}(0)\left(1+\delta \sin ^{2}(\theta) \cos ^{2}(\theta)+\varepsilon \sin ^{4}(\theta)\right)
$$

In Fig. 3, the anisotropic parameters are greatest where the logs show the most variance over the $3 \mathrm{~m}$ averaging interval. They indicate only the layering component of the anisotropy since we have no data on the intrinsic anisotropy of the basalt. However, delta is small with a mean of zero while epsilon is positive with most values less than 0.05 and almost all values less than 0.1 . We found a similar range of epsilon and delta when the averaging interval was increased to $10 \mathrm{~m}$. This means that the modelled effect of layering is to produce horizontal compressional wavespeeds around 5\% faster than vertical wavespeeds, a prediction which contradicts the observation by Kiørboe $\&$ Petersen (1995) of vertical compressional wavespeeds being about $10 \%$ higher than horizontal compressional wavespeeds in the upper $800 \mathrm{~m}$ of the basalt beds. Kiørboe \& Petersen (1995) appeal to vertical fractures associated with columnar basalts and master joints to explain their observation, a point which we discuss below.

\section{Azimuthal anisotropy direction}

Aligned vertical fractures would be expected to result in azimuthal anisotropy. To test for this in Lopra-1/1A, the dipole shear sonic tool was logged in four-component mode whereby both of the two orthogonal dipole receiver arrays recorded signals from each of the two orthogonal dipole sources, energised sequentially. The data were processed for the presence of fast and slow shear waves corresponding to shear propagation along the borehole with polarisations parallel and perpendicular to the assumed fractures. One output of the azimuthal processing is a log of the azimuth of the fast shear wave. The direction of the fast shear wave is found by determining the shear waveform rotation which minimises the cross-energy, for example the energy recorded by the Y-polarised receiver from the X-polarised source (Esmersoy et al. 1994), then picking the faster of the two shear estimates. The difference in cross-energy between its maximum and minimum excursions as a function of rotation angle is a measure of reliability of the anisotropy estimate. In Figs $4 \mathrm{a}$ and $4 \mathrm{~b}$ we plot the fast shear direction results in different depth intervals, where the vector from the centre of the plot to a given depth point has an azimuth corresponding to that of the fast shear polarisation and a magnitude corresponding to the cross-energy difference normalised by the total energy. In order to display only the most reliable estimates of the fast shear direction, the estimates were windowed according to cut-off values of calliper reading, the estimated error in azimuth, the anisotropy estimate and the normalised cross-energy difference (cut-offvalues indicated on the figure). The plots are radially symmetric through the origin because of the $180^{\circ}$ ambiguity in determining azimuth. In Fig. 4a, from 511-540 m KB, we see that there is a well-defined fast shear direction at $\mathrm{N} 31^{\circ} \mathrm{E}$, broadly consistent with the NE-SW strike of one of the two mapped master joint sets (Kiørboe \& Petersen 1995, fig. 1). Below $540 \mathrm{~m} \mathrm{~KB}$, the fast shear direction rotates through $100^{\circ}$ to $\mathrm{N} 131^{\circ} \mathrm{E}$, al though this direction is less well defined. In Fig. 4b, there is a fairly consistent fast shear direction of about $\mathrm{N} 144^{\circ} \mathrm{E}$ in the interval $2991-3502 \mathrm{~m} \mathrm{~KB}$, but with a larger scatter in azimuth. The cross-energy cut-off value in Fig. $4 \mathrm{~b}$ has been reduced, compared to Fig. 4a, to capture more estimates. The estimates of the fast shear direction deeper than $540 \mathrm{~m} \mathrm{~KB}$ are consistent with the NW-SE strike of the second mapped joint set in Kiørboe $\&$ Petersen's (1995) fig. 1.

From the earlier VSP and refraction profile data, Kiør- 
Fig. 4. a: Fast shear direction estimated from the dipole shear log over the depth interval 511-1010 $\mathrm{m} \mathrm{KB}$. The log was recorded in four component mode and rotated to minimise the cross-energy. Two orthogonal directions are evident. The better-defined direction is over a fairly short interval from 511-540 m $\mathrm{KB}$, consistent with one of the mapped master joints, and parallel to the offset seismic surveys described in Kiørboe \& Petersen (1995). b: Fast shear direction estimated over the interval 2991$3530 \mathrm{~m} \mathrm{~KB}$. The fast direction is less well defined than in the shallower interval but is consistent with mapped joints.
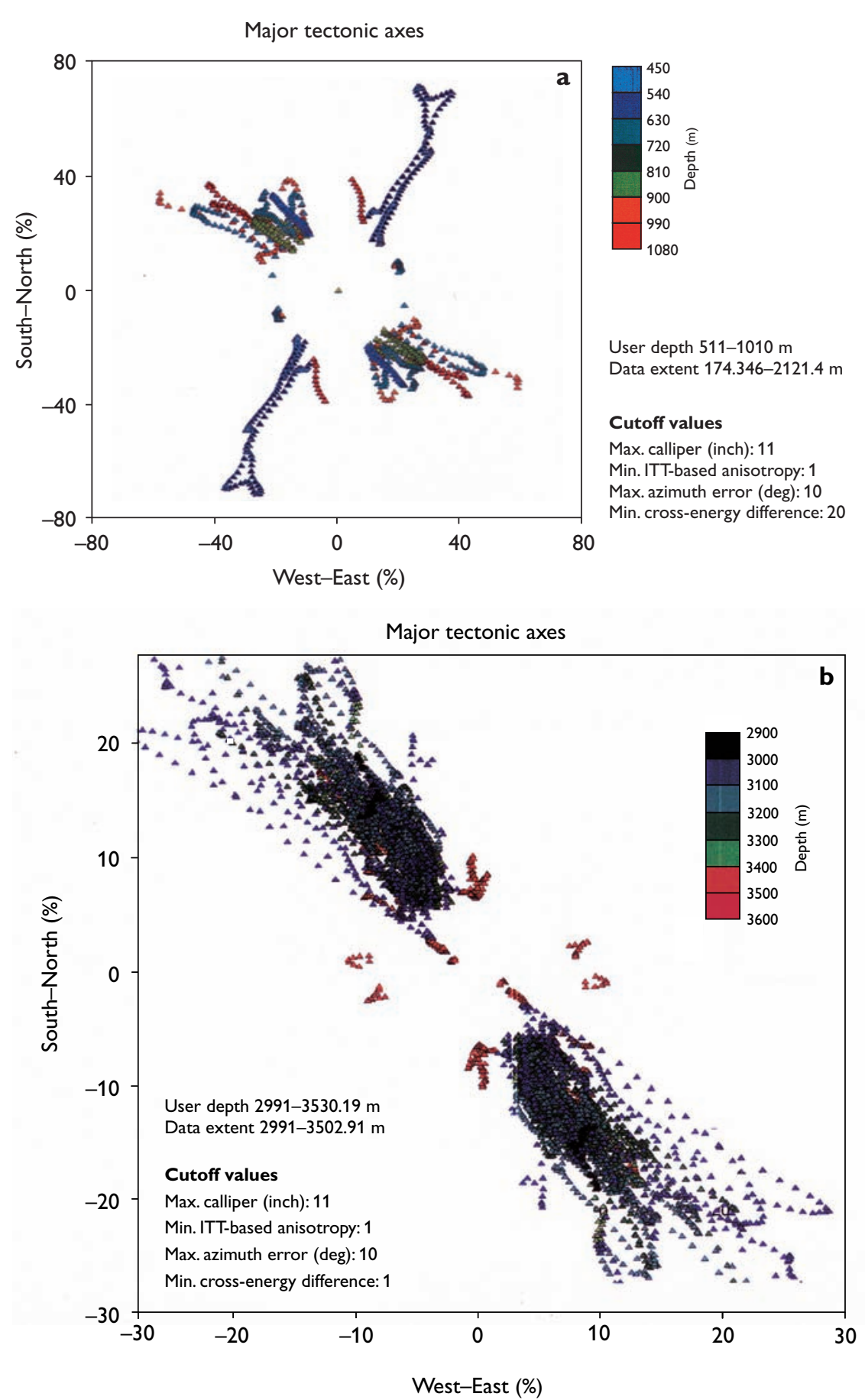

boe \& Petersen (1995) reported vertical $V_{P}$ values about $10 \%$ higher than horizontal $V_{P}$ in the upper 800 m of the section around the well and suggested that a combination of vertical, columnar fractures and NW-SE master joints crossing the ray-paths in the refraction profile may be responsible. The present analysis shows little azimuthal anisotropy on the dipole shear $\log$ and, while the fast shear direction appears to be $\mathrm{N} 131^{\circ} \mathrm{E}$ below $540 \mathrm{~m} \mathrm{~KB}$, there is a well-established, fast shear direction of $\mathrm{N} 31^{\circ} \mathrm{E}$ from 511-540 m KB. If the shear azimuthal anisotropy results from fractures or stress, then the compressional wavespeed anisotropy should follow the same directions. Formation microscanner data from the Lopra Deepening Project show the presence of sub-horizontal conductive features, suggestive of horizontal fractures, although there is a question as to whether these are natural or drilling induced. The only conventional core (2380 m KB) shows cemented, horizontal fractures (L. Kiørboe, personal communication 1996) but few vertical fractures were observed, consistent with the weak azimuthal anisotropy observed in 


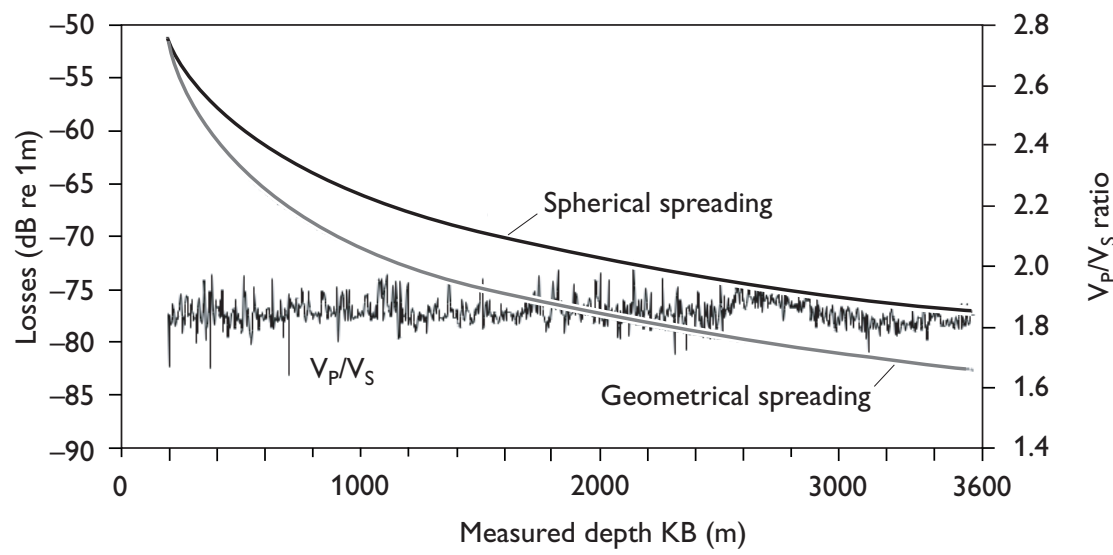

Fig. 5. Plot of logged $V_{\mathrm{P}} / \mathrm{V}_{\mathrm{S}}$ ratio and short-offset geometrical spreading computed using Newman's (1973) relation for both the logged velocities and a constant velocity medium. $3 \mathrm{~m}$ averaged logs.

the dipole shear log. In addition, the layer-induced anisotropy modelled by upscaling the log data suggests that horizontal compressional wavespeeds should exceed vertical wavespeeds by around 5\%. Can we resolve the apparent contradiction? Possible explanations include: (1) the high-wavespeed, dolerite intrusions seen on the logs down to $850 \mathrm{~m} \mathrm{~KB}$ affect the vertical velocity locally; (2) the layering-and fracture-induced anisotropy revealed by the well logs is not representative of the volume sampled by the VSP and earlier refraction profile; (3) the unreversed refraction profile sees low apparent velocities because of the basalt flows that dip $3-7^{\circ}$ to the north-east; and (4) there is a mis-correlation of events between the refraction profile and the earlier VSP. Kiørboe \& Petersen (1995) reported that a $700 \mathrm{~m}$ refraction profile shot towards the WNW from the 1988 VSP source point through the well yielded an apparent $V_{P}$ of $4.8 \mathrm{~km} / \mathrm{s}$, the same as that observed over the offset range $2.3-14 \mathrm{~km}$ on the long refraction profile. The azimuth differences of these two profiles weakens the case that structural dip or aligned master joints affect the velocities. That the fractures in the core sample are cemented also discounts the possibility that open, vertical, hexagonal fractures are prevalent, although we cannot disprove this possibility because of the small volumes sampled by core and borehole logs. Our preferred explanation is that the geometry of the dolerite intrusions increases locally the estimate of the vertical velocities at the well. Further support for this hypothesis comes from the well-tie, described below, where there is evidence that the VSP sees a higher velocity than the sonic tool in the shallow section, possibly due to the dolerite intrusions providing a VSP ray-path away from the borehole that is faster than that seen by the sonic tool along the borehole. Kiørboe \& Petersen (1995) also found that the velocities derived from the 1988 VSP were too fast to simulate the arrival times on the longer offset data.

\section{Log-derived seismic characteristics and well-tie}

In this section we develop further the seismic properties of the stacked basalt flows in Lopra-1/1A as modelled from the log data. Figure 5 displays the $\mathrm{V}_{\mathrm{P}} / \mathrm{V}_{\mathrm{S}}$ ratio and the normal incidence, two-way geometrical spreading, computed using Newman's (1973) relation:

$$
D_{t w o-w a y}=2 \frac{\sum d_{i} V_{i}}{V_{1}}
$$

for the $3 \mathrm{~m}$ Backus-averaged data since there is no expected dependency on sample interval. In this relation, $D_{t w o-}$ way is the two-way loss, relative to the amplitude at $1 \mathrm{~m}$ from the source, of a seismic wave propagating down through a 1D stack of $i$ layers, each of thickness $d_{i}$ and interval velocity $V_{i}$, and back to the surface again. $V_{1}$ is the velocity of the first layer and in this case is taken to be $2792 \mathrm{~m} / \mathrm{s}$. Because the thickness of the superficial layer between the source and the top of the sonic log is $177.67 \mathrm{~m}$, the two-way loss at the top of the sonic log is:

$$
\mathrm{D}_{\text {two-way }}=-20 \log (2 \times 177.67)=-51.01 \mathrm{~dB} \text { re } 1 \mathrm{~m}
$$

Figure 5 shows the modelled geometrical spreading in the basalt flows, compared to the spherical loss in a uniform medium for reference. The extra two-way geometrical spreading due to the velocity variations is around $5.5 \mathrm{~dB}$ for a reflector at the total depth of the well, resulting in a spreading loss of $82.5 \mathrm{~dB}$ re $1 \mathrm{~m}$.

The $V_{P} / V_{S}$ ratio from the $3 \mathrm{~m}$ averaged logs (Fig. 5) is almost constant over the well at 1.85 , with a standard deviation of 0.04 . We also cross-plotted the log values of $V_{P}$ and $V_{S}$, upscaled to $1 \mathrm{~m}$ to reduce fluctuations, using a Backus average. Figure 6 shows the cross-plot of $V_{P}$ to $V_{S}$ with a histogram of each variable indicating the number of data points falling into velocity bins of $100 \mathrm{~m} / \mathrm{s}$ for $V_{P}$ 
Fig. 6. $\mathrm{V}_{\mathrm{P}}-\mathrm{V}_{\mathrm{S}}$ cross-plot of $1 \mathrm{~m}$ Backusaveraged logs with histograms and linear regression. The histograms show the frequency distributions of $V_{P}$ and $V_{S}$ data gathered in bins of $100 \mathrm{~m} / \mathrm{s}$ and $55 \mathrm{~m} / \mathrm{s}$ respectively, to maintain the same number of bins for each variable. The regression yields a $V_{\mathrm{P}} / \mathrm{V}_{\mathrm{S}}$ ratio of 1.84 with a standard deviation of 0.01 . The similarity of the histograms supports the choice of a linear regression.

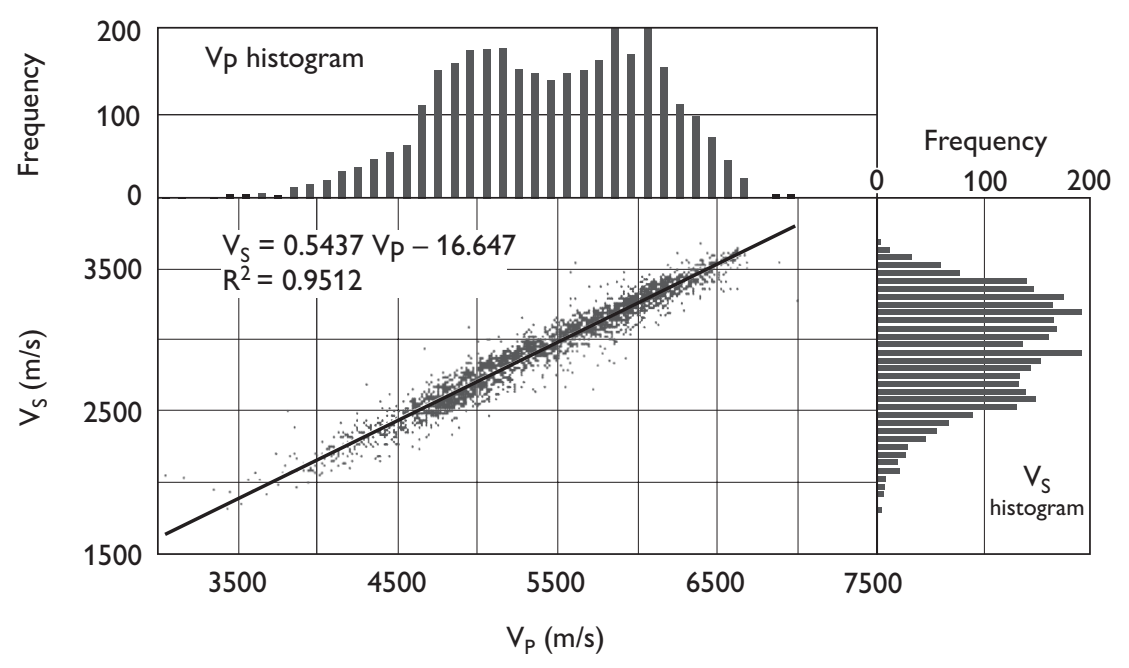

and $55 \mathrm{~m} / \mathrm{s}$ for $\mathrm{V}_{\mathrm{s}}$. The bin-widths are scaled approximately by the $V_{\mathrm{P}} / V_{\mathrm{S}}$ ratio, for which a value of 1.84 was obtained by linear regression with a standard deviation of 0.01 . The similarity of the histograms supports the linearity of $V_{P}$ and $V_{S}$ over such a large range of values. This linearity is consistent with the observation of Planke \& Cambray (1998) who studied subaerially emplaced lava piles sampled in Hole 917A of the Ocean Drilling Programme. The absence of a trend, with the possible exception of the hyaloclastite interval from $2600-2850 \mathrm{~m} \mathrm{~KB}$, suggests that there is no significant compaction effect with depth.

We computed two-way, plane-wave, transmission losses for the $15.24 \mathrm{~cm}$ logs as well as for logs Backus-averaged to $30 \mathrm{~cm}, 50 \mathrm{~cm}, 1 \mathrm{~m}, 3 \mathrm{~m}, 5 \mathrm{~m}$ and $10 \mathrm{~m}$ (actually, the nearest multiple of $15.24 \mathrm{~cm}$ to these values). The transmission losses are the $1 \mathrm{D}$, ray-theoretical loss from a unit amplitude plane-wave source pulse which is partially transmitted and reflected from each interface in its twoway path from the surface to a given reflector and back to the surface again. It is computed from the well-known relation

$$
T L=\prod\left(1-\mathrm{R}_{\mathrm{i}}^{2}\right)
$$

where $R_{i}$ is the plane-wave, normal-incidence, reflection coefficient at the $i$-th boundary

$$
R_{\mathrm{i}}=\frac{\rho_{i+1} V_{p i+1}-\rho_{i} V_{p i}}{\rho_{i+1} V_{p i+1}+\rho_{i} V_{p i}}
$$

and where $\rho_{i}$ and $V_{P i}$ are the density and compressional wavespeed of the $i$-th layer. In this simple model, there is no intrinsic loss. Instead, the loss is due to progressive scattering and consequent removal of energy from the firstarriving, primary pulse. The results are displayed in Fig. 7 along with the $\mathrm{V}_{\mathrm{P}} \log$ for correlation. Losses over the full interval vary non-linearly from $15 \mathrm{~dB}(10 \mathrm{~m} \log )$ to $73 \mathrm{~dB}$ $(50 \mathrm{~cm} \mathrm{log})$, because of the non-linear dependence on the reflection coefficient. The highest loss is seen at the $50 \mathrm{~cm}$ sampling. As suggested by O'Doherty \& Anstey (1971), an impedance gradient represented by a single large reflection coefficient has more effect on the transmission losses than several smaller coefficients, so very fine sampling $(15 \mathrm{~cm})$ can produce a smaller loss than the upscaled logs, as seen here. However, blocking intervals larger than bed thicknesses progressively fail to represent beds at all and so will predict less loss. While there is the expected strong dependency of transmission losses with sample interval, all the curves show broadly similar features, with the lower contrast, hyaloclastite interval below $2600 \mathrm{~m}$ $\mathrm{KB}$ exhibiting reduced loss compared to the section of high contrast flow units, which resembles the cyclic example in O'Doherty \& Anstey (1971) and which is expected to have a high scattering loss.

The combined effects of modelled normal-incidence spreading and plane wave transmission losses range from $97.5 \mathrm{~dB}$ to $155.5 \mathrm{~dB}$ re $1 \mathrm{~m}$, where the wide margin results from the uncertain effects of the transmission losses. We shall return to this point in the analysis of the VSP amplitudes. However, we emphasise that the loss estimates here are those due to near-normal propagation in a $1 \mathrm{D}$ medium. As offset increases, geometrical losses increase rapidly due to refraction effects at high velocity contrast boundaries. Similarly, transmission coefficients vary strongly with incidence angle at high contrast boundaries. The cone of forward propagation of the compressional wave is limited by critical angle effects and offers opportunities for mode conversions to be observed over a wider range of incidence angles and offsets.

The VSP first arrival travel times were picked to derive 


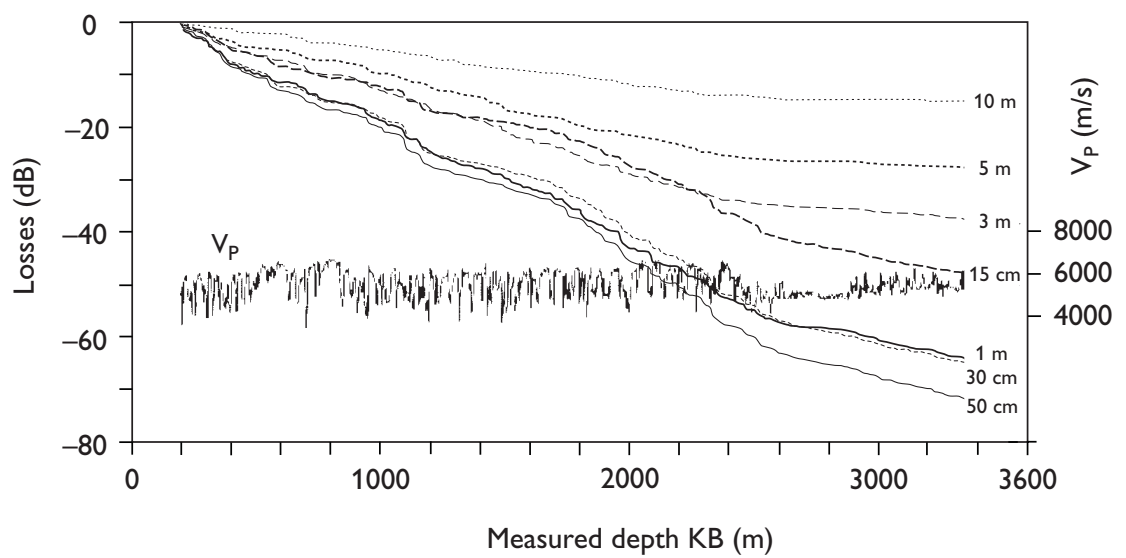

Fig. 7. Modelled plane-wave, normalincidence, two-way transmission losses as a function of log sampling, together with $\mathrm{V}_{\mathrm{P}} \log$ for correlation. Losses are nonlinear with sampling but show generally similar behaviour with high contrast layering resulting in the greatest scattering loss. a seismic two-way time to true vertical depth curve. The time-depth curve from the VSP was used to 'calibrate' the TVD-corrected sonic log, provide a fine-scale, seismic time-depth interpolation and to generate a synthetic seismogram. A drift curve was computed by integrating the compressional slowness log between the VSP time-depth pairs and taking the difference between the VSP and sonic interval times. We observed positive drift (VSP interval time greater than sonic) over most of the logged interval. This implies a lack of environmental effects on the sonic (which usually increase the sonic slowness) leaving the normal dispersion effect between seismic and sonic frequencies as the dominant mechanism. Negative drift (sonic interval time greater than VSP) was seen in the shallow part of the well, above $800 \mathrm{~m} \mathrm{~KB}$. This would be normal in a sedimentary sequence, indicating a washed-out hole or altered, unconsolidated formations. However, in the basalt environment, formation alteration is unlikely; even though the well shallower than $800 \mathrm{~m} \mathrm{~KB}$ is often out of gauge, it is not different from the rest of the borehole drilled in 1981 which displays positive drift. Possible explanations of the negative drift are unreliable VSP picks due to shallow, unsupported casing interference (the compressional wavespeed of the formation is similar to that of the casing extensional mode), or possible refraction along the high velocity $(6.5 \mathrm{~km} / \mathrm{s})$ dolerite intrusions. If the VSP wave front refracts along the intrusions such that the borehole does not form the VSP raypath, then the VSP interval time will be less than the sonic interval time. In any case, the picks above $800 \mathrm{~m}$ have been interpreted in such a way as to avoid undue, and possibly unrealistic, correction to the sonic.

The corrected compressional sonic log was used to convert the shear sonic and the density logs from a common depth scale to a common two-way time scale which is assured to match that of the VSP. Note that this implicitly changes the $V_{\mathrm{P}} / \mathrm{V}_{\mathrm{S}}$ ratio, since the VSP was not used to correct the shear sonic slowness values. Deeper than $800 \mathrm{~m}$, the compressional slowness drift is about $15 \mathrm{~ms}$ in $2200 \mathrm{~m}$, or $6 \mu \mathrm{s} / \mathrm{m}$. However, the average interval slowness is about $190 \mu \mathrm{s} / \mathrm{m}$, so the drift correction is reasonably significant at $3.4 \%$ in slowness. Care was taken in choosing the correction points to avoid introducing false reflection events.

The time-based logs drove a 1D model of equal timethickness layers. The resulting primaries-only, reflection coefficient sequence without transmission losses was convolved with a $40 \mathrm{~Hz}$, zero-phase, Ricker wavelet to create a synthetic seismogram. The synthetic was spliced into the VSP up-going wavefield along the two-way time-depth curve to facilitate event correlation (the right-hand panel of Fig. 8). The VSP has been waveshaped to a zero phase wavelet of bandwidth $10-70 \mathrm{~Hz}$ and both the synthetic and VSP traces are displayed in reverse SEG polarity, so that an increase in acoustic impedance with depth is displayed as a black peak. Figure 8 shows the correlation from a Two-Way Time (TWT) of $650 \mathrm{~ms}$, where several events can be seen to tie in time and in character, resulting in an unambiguous correlation. In an igneous province, a tie of this quality is relatively unusual and suggests that the lateral variability of the basalt flows is mild, at least over the extent of a VSP Fresnel zone which is several tens to hundreds of metres, depending on the elevation of the VSP tool above the reflector. Residual ringing from the uncemented casing is visible in the up-going VSP wavefield on the right edge of the section.

\section{Correlations from the well-tie}

Using Fig. 8 we discuss the correlation to the lithological summary taken from the Lopra-1/1A End of Well Report (EWR), subsequently modified by R. Waagstein (personal communication 2001). The displayed interval from 650-1430 ms TWT shows $V_{\mathrm{P}}, \mathrm{V}_{\mathrm{S}}$, density and Poisson's 


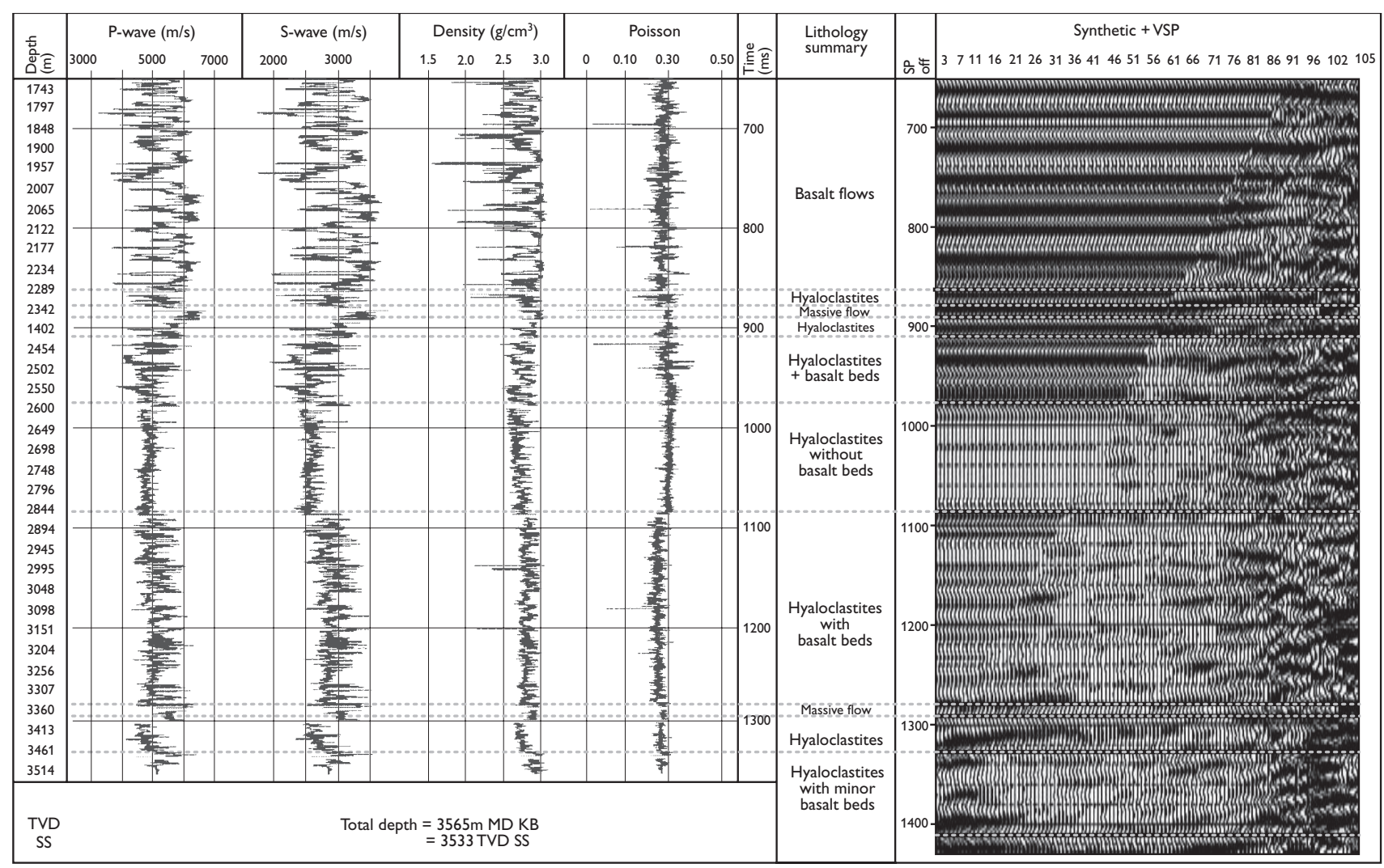

Fig. 8. Correlation of the VSP up-going wavefield with the log-derived, reflection coefficient sequence convolved with a $40 \mathrm{~Hz}$ zero-phase Ricker wavelet. The VSP wavefield has been waveshaped to zero phase over the bandwidth 10-70 Hz. The VSP and synthetic are correlated with the time-based logs and lithostratigraphy (R. Waagstein, personal communication 2001) as listed in Table 1.

Ratio logs. At the left edge of the display is a non-linear scale in True Vertical Depth extending from $1714 \mathrm{~m}$ to TD at $3533 \mathrm{~m}$ TVDSS, which corresponds to $3565 \mathrm{~m}$ KB. Horizontal lines mark particular correlations between the well logs, the lithology summary, the synthetic seismogram and the VSP. These correlations are summarised in Table 1 and further comments relating to the pre-drill targets are made below.

From 882-912 ms, the VSP shows a package of strong reflections, with a white trough at $894 \mathrm{~ms}$, seen on the pre-drill VSP at $920 \mathrm{~ms}$ and identified as one of the target horizons for the well. This event was prognosed by Kiørboe \& Petersen (1995) at $2.34 \mathrm{~km}, 162 \mathrm{~m}$ below the original TD of Lopra- 1 and interpreted as a decrease in impedance. In fact, the reflection package comprises at least two near-tuning events. A sharp increase in impedance at $882 \mathrm{~ms}$ (2361 m KB, $2345 \mathrm{~m}$ TVDSS) corresponds to the top of a massive basalt flow that gives rise to a black peak on the VSP. The trough associated with the sidelobe to this wavelet reinforces a broad, weak trough caused by the decrease in impedance at the base of the flow around $894 \mathrm{~ms}$ (2401 m KB, $2385 \mathrm{~m}$ TVDSS), which reflects the highly amygdaloidal top of an underlying flow. The reflection package is seen more strongly on the VSP than on the synthetic seismogram, possibly due to lateral heterogeneity near the well.

Another package of strong events was drilled above TD from 1274-1340 ms. During drilling, it was hoped that these reflections, seen at $1350 \mathrm{~ms}$ on the pre-deepening VSP (Kiørboe \& Petersen 1995), might mark either siliciclastic sediment or basement. However, the sharp drop in impedance at $3427 \mathrm{~m} \mathrm{~KB} \mathrm{(3397} \mathrm{m} \mathrm{TVDSS)} \mathrm{at} \mathrm{the} \mathrm{base}$ of a thick, massive basalt bed results in the trough at 1294 ms seen on both the VSP and the synthetic, which correlates with another hyaloclastite sequence with a thin tuff at the top (R. Waagstein, personal communication 2001). Another drop in impedance was drilled at $3512 \mathrm{~m}$ KB (3480 m TVDSS), which also corresponds to a strong white trough at $1328 \mathrm{~ms}$ on both the synthetic and the VSP. It probably corresponds to the event at $1350 \mathrm{~ms}$ seen on the pre-drill VSP. It displays moveout to earlier times with decreasing geophone depth, indicating dip of the beds.

Just below TD, at $1412 \mathrm{~ms}$ on the VSP, is a persistent, large-amplitude, symmetric, white trough, which may be 


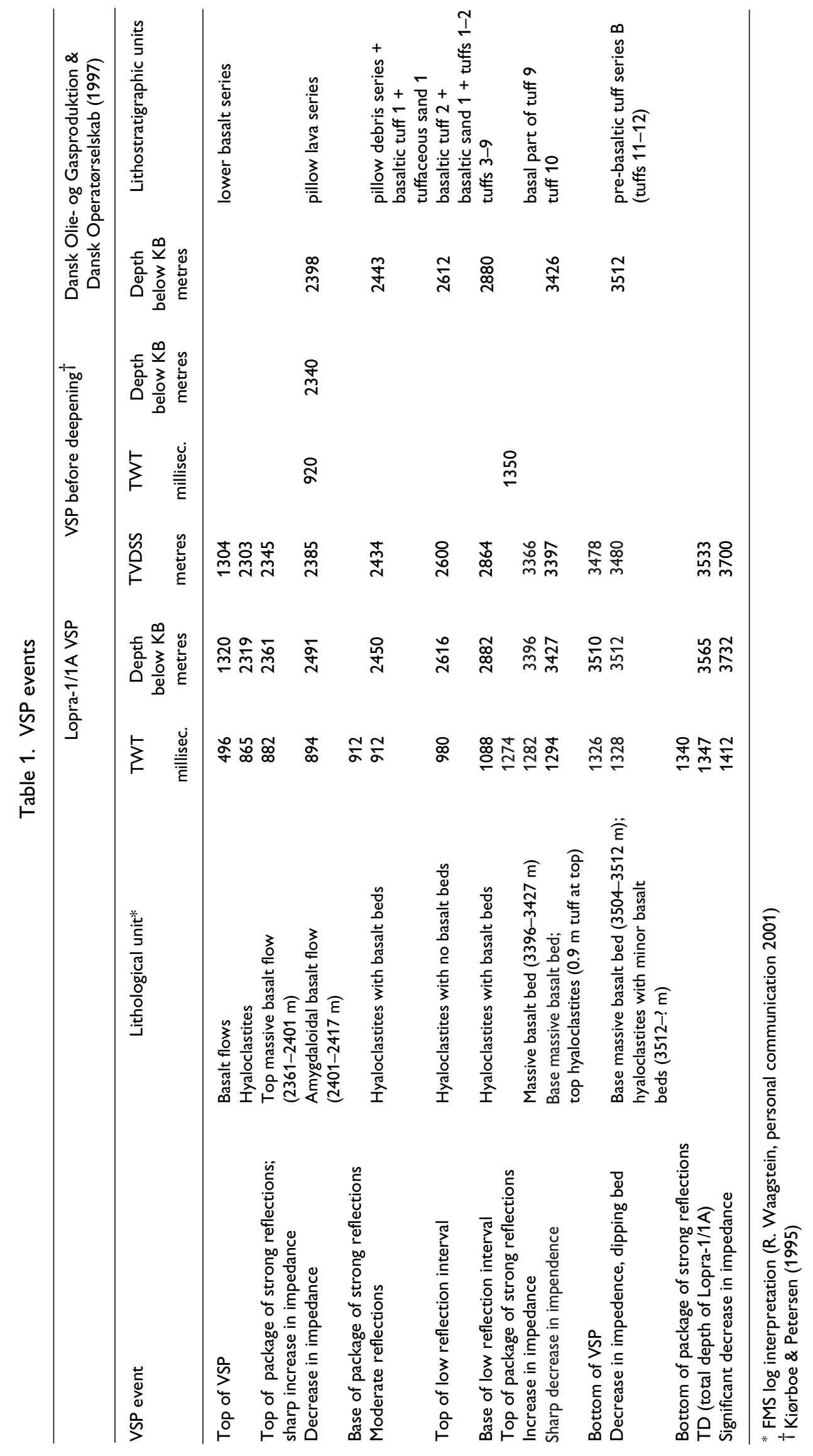




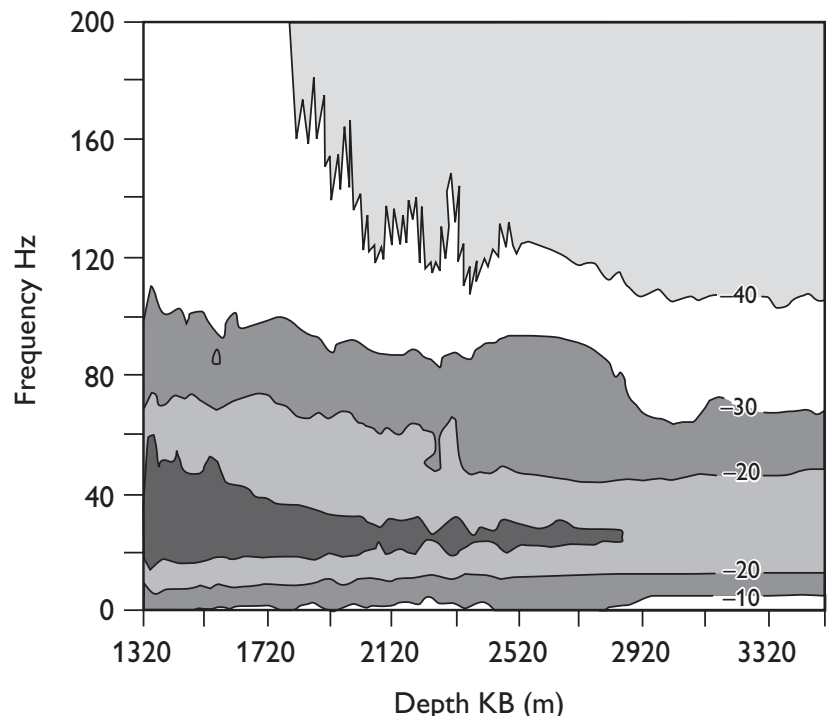

Fig. 9. Contoured power spectrum of the energy in a $150 \mathrm{~ms}$ window around the down-going first arrivals in the VSP. The upgoing wavefield was removed first. The contour levels are $\mathrm{dB}$ down from the peak value.

interpreted as a significant drop in impedance. This is about $86 \mathrm{~ms}$, or $222 \mathrm{~m}$, below the deepest VSP level at $3510 \mathrm{~m} \mathrm{~KB}(3478 \mathrm{~m}$ TVDSS) and therefore $167 \mathrm{~m}$ below the final TD of $3565 \mathrm{~m} \mathrm{~KB}$ (3533 m TVDSS), assuming an extrapolation velocity of $5150 \mathrm{~m} / \mathrm{s}$. The event is as tantalising as that seen at $920 \mathrm{~ms}$ on the pre-deepening VSP, which was drilled some $162 \mathrm{~m}$ below the original TD of Lopra-1. However, similar, though weaker, 'soft kicks' seen on the seismic data have turned out to be due to contrasts within the volcanic pile and the reflection at $1412 \mathrm{~ms}$ TWT is unlikely to be basement, so it is probable that more volcanic sequence lies below the present TD. In contrast with the event at $1328 \mathrm{~ms}$, this strong arrival below TD shows little moveout and suggests low dip.

\section{VSP loss estimates and amplitude modelling}

As noted above, ray-theoretical estimates of transmission losses are strongly dependent on the log sampling, so we derived loss estimates from the VSP downwave before turning to full waveform modelling to simulate the observed propagation effects. The contoured power spectrum by depth is shown in Fig. 9, after windowing the first $150 \mathrm{~ms}$ of data from the $2 \mathrm{~ms}$ sampled downgoing wavefield. Two points are apparent: (1) there is a low-frequency roll-off to the data caused by the start frequency of the vibrator sweep at $10 \mathrm{~Hz},(2)$ there is a smooth decline in frequency content with depth.

We estimated the root mean square (RMS) amplitudes within a window of $150 \mathrm{~ms}$ about the VSP first breaks, corrected the amplitudes for geometrical spreading using the spreading loss curve displayed in Fig. 5 and plotted the results in Fig. 10 together with the ray-theoretical transmission losses at $3 \mathrm{~m}, 5 \mathrm{~m}$ and $10 \mathrm{~m}$ sampling. Since the amplitude of the VSP top level is arbitrary, we matched the slopes of the transmission loss curves by eye. The VSP amplitude decay curve, after spreading correction, shows a character similar to the modelled transmission losses, although, given our earlier comments on the unreliability of transmission losses, the match to the transmission loss curve at $5 \mathrm{~m}$ sampling is probably coincidental. However, the change in slope around $2500 \mathrm{~m} \mathrm{~KB}$ on the modelled curves, which is due to the transition from high contrast basalt beds to low contrast hyaloclastites, is also evident on the VSP. By reciprocity, the transmission seismogram going back up through the basalt sequence is the same as that going down through the sequence. Hence, the reflection seismogram should be the time-delayed, one-sided correlation of the down-going wave. Strictly speaking, the two-way loss estimates should be made after convolving
Fig. 10. Losses from RMS amplitudes estimated in $150 \mathrm{~ms}$ window around the first arrivals in the VSP, after correcting for geometrical spreading using the logderived spreading loss estimates from Fig. 5. The first VSP level amplitude is arbitrary and has been selected visually to overlie the upscaled log curve of modelled transmission losses with the most similar slope. Regression lines have been fitted over the VSP intervals indicated to estimate effective $\mathrm{Q}$ for a wavelet dominant frequency of $36 \mathrm{~Hz}$.

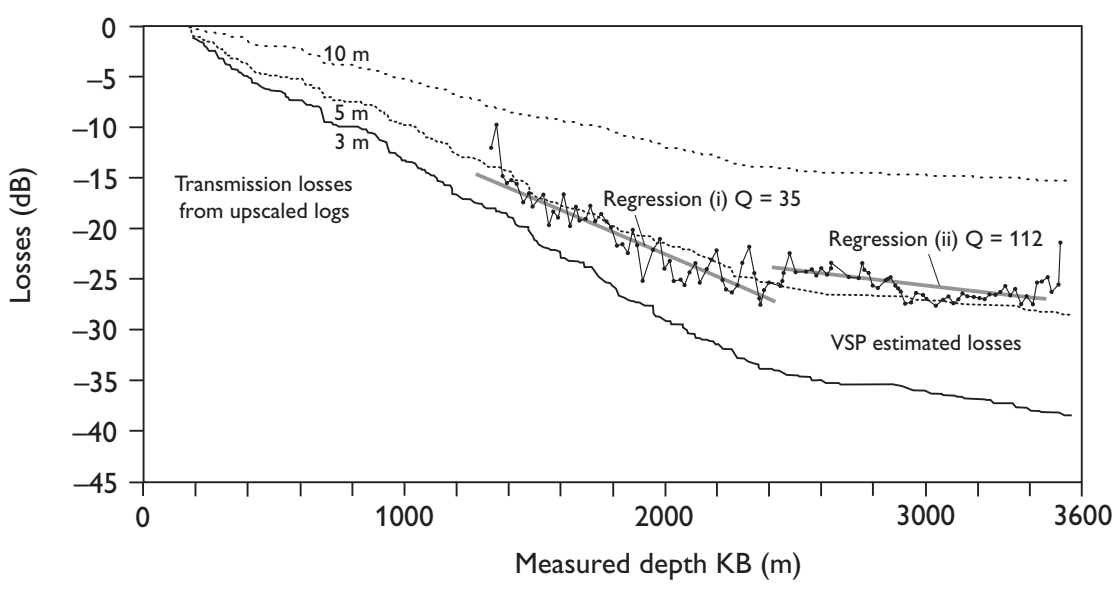


the down-going wave with itself. Since the amplitude effect of cascaded filters is to take the product of their gain functions, we have approximated the amplitude effect of the two-way propagation by doubling the $\mathrm{dB}$ loss estimated from the one-way measurement of RMS time-domain amplitudes.

Because of the change in slope of the transmission loss curves around $2500 \mathrm{~m} \mathrm{~KB}$, we estimated two effective Q factors to represent the scattering loss. Over the interval of periodic layering, from $1350 \mathrm{~m} \mathrm{~KB}$ to $2450 \mathrm{~m} \mathrm{~KB}$, we fitted a linear trend and estimated losses corresponding to an effective Q of 35 for a dominant period wavelet of $27.5 \mathrm{~ms}$. The $95 \%$ confidence limits on the regression map to a range in effective $Q$ of 32 to 40 .

From $2450 \mathrm{~m} \mathrm{~KB}$ to $3510 \mathrm{~m} \mathrm{~KB}$, the loss curve is reduced and a linear regression resulted in a loss corresponding to an effective $Q$ in excess of 100 . The inverse correlation of effective $Q$ values with the change in impedance contrasts further supports the inference that the volcanic sequence has low intrinsic loss and that its attenuation is principally due to scattering. This is consistent with the results of other, similar studies (e.g. Pujol \& Smithson 1991) which found that spreading and scattering losses could account for observed VSP amplitude behaviour in basalt sequences. Evidently, we can find a log sample rate such that the ray-theoretical losses match the VSP loss estimates. However, given the large variability in the transmission losses with sampling, we undertook further analysis to test the hypothesis that scattering loss is the dominant mechanism.

We estimated spectral ratios of windowed VSP downwave traces at $2420 \mathrm{~m}, 2980 \mathrm{~m}, 3044 \mathrm{~m}$ and $3500 \mathrm{~m} \mathrm{~KB}$, using the downwave at $1880 \mathrm{~m} \mathrm{~KB}$ as a reference. The spectral ratios are plotted in Fig. 11 with the amplitude spectrum of the reference level. Over the rather limited frequency interval of the strongest signal $(17-40 \mathrm{~Hz})$, the slopes of the spectral ratios are effectively the same and rather flat. In a lossy medium, the slopes are modelled by

$$
-\frac{20 \pi \Delta z}{Q c} \log _{10} e
$$

where $c$ is the average velocity over the interval $\Delta z$. The four slopes in Fig. 11 correspond to depth intervals of $548 \mathrm{~m}, 1100 \mathrm{~m}, 1164 \mathrm{~m}$ and $1620 \mathrm{~m}$, but there appears to be little variation of slope with the depth interval. The real VSP amplitude loss is not well modelled by frequencydependent attenuation.

Nevertheless, the variation in spectral power with depth in Fig. 9 does show a loss of high frequencies with depth, mainly in the shallower part of the section, with little apparent bandwidth change over much of the deeper part of the volcanic sequence. We therefore modelled the propagation of a VSP pulse generated at ground level (GL) through a $185 \mathrm{~m}$ uniform layer on top of the pile of basalt flows logged from $185 \mathrm{~m}$ below GL and emerging into a uniform half-space at $2160 \mathrm{~m}$ below GL (Fig. 12). The $1975 \mathrm{~m}$ interval was modelled using a full-elastic, 1D modelling code based upon the Kennett algorithm (Kennett 1974, 1983) and developed at Schlumberger Cambridge Research. The $15 \mathrm{~cm}$ log data were upscaled to $3 \mathrm{~m}$ by Backus averaging to reduce the computational cost. Equivalent medium averaging, using windows of approximately $1 / 20$ th of the dominant seismic wavelength, provides a convenient method of upscaling logged data to allow efficient elastic waveform modelling, while retaining fidelity of both the travel time and amplitude information of the log scale model (Folstad \& Schoenberg 1992, 1993).

Propagation was modelled both with and without multiple scattering, with 3D geometrical spreading from a point source. The injected wavelet at the top of the stack is a zero-phase, $60 \mathrm{~Hz}$ Ricker wavelet. (The wavelet appears not to be zero phase because of near field effects.) At the base of the stack, the escaping wavelet modelled without multiples is zero phase, but its amplitude has been diminished, undergoing a one-way loss of $46.9 \mathrm{~dB}$ due to

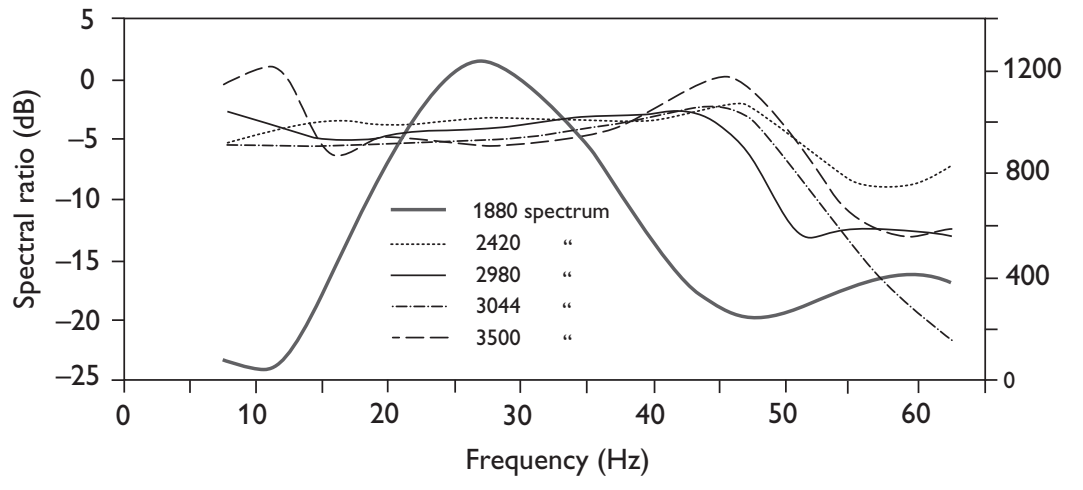

Fig. 11. Spectral ratios computed from four VSP levels relative to a reference level at $1880 \mathrm{~m} \mathrm{~KB}$. The amplitude spectrum of the reference trace is also shown to indicate the signal frequency band. 
Fig. 12. Full elastic model of VSP downwave propagation through a stack of $3 \mathrm{~m}$ layers derived from the Backus average of the $V_{P}, V_{S}$ and density logs between $185 \mathrm{~m}$ below ground level (GL) and $2160 \mathrm{~m}$ below GL. Propagation was modelled with and without peg-leg multiples and amplitudes were estimated in a $40 \mathrm{~ms}$ window about the wavelets.
One-way loss (geometrical spreading and transmission losses with multiples) $=37.5 \mathrm{~dB}$
One-way loss (geometrical spreading and transmission losses without multiples) $=46.9 \mathrm{~dB}$

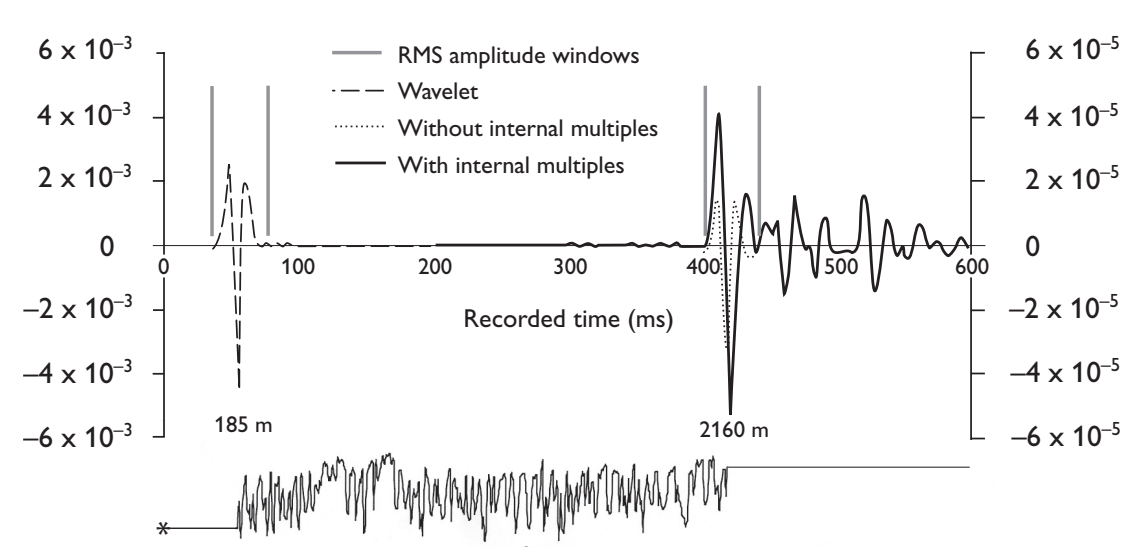

Point source

Velocity model the combination of one-way geometrical spreading and transmission losses. The escaping wave modelled with all internal multiples has been phase rotated within the $40 \mathrm{~ms}$ analysis window and has a higher RMS amplitude than the wavelet propagated without multiples, because shortperiod multiples have served to boost amplitudes within the analysis window. The RMS amplitude of the escaping wave with multiples is $-37.5 \mathrm{~dB}$ relative to the input wavelet at the top of the stack. However, energy has been removed from the front of the wavelet, its trough-to-trough duration has increased and a coda has developed. These effects are due to scattering by the high-contrast layering.

We ran a further model, with multiples, to study the frequency-dependent effects of multiple scattering by the periodic layering by using a wide-band source signature defined in the frequency domain from DC to the Nyquist frequency at $125 \mathrm{~Hz}$, corresponding to $4 \mathrm{~ms}$ sampling (in contrast to the real VSP which was acquired at $2 \mathrm{~ms} \mathrm{sam}$ pling). We also altered the model by adding a lower halfspace simulating a massive sand unit and placing the deeper receiver at $300 \mathrm{~m}$ into the sand below the base basalt which was again modelled at $2160 \mathrm{~m}$.

The Fourier domain amplitudes of the deep- and shallow-receiver down-going compressional waves are displayed in Fig. 13a, where the low-pass filtering effect of the basalt sequence can be seen, with quite deep notches appearing from $39 \mathrm{~Hz}$, although higher frequency peaks also appear, such as that at $85 \mathrm{~Hz}$.

The spectral ratios of the wide-band synthetics are shown in Fig. 13b with a linear regression to $80 \mathrm{~Hz}$, avoiding the higher frequency side-lobes. The regression pro- vides an effective Q estimate of 32, with 95\% confidence limits of 26 and 42, which is consistent with the time domain estimate of 35 from the real VSP over the interval of basalt flows shown in Fig. 10. However, we note that while the real VSP displayed little or no frequencydependent loss over the interval from 1880-3500 m KB, the full elastic modelling does support the assertion that the amplitude loss can be modelled by scattering and geometrical spreading alone.

\section{Loss estimates from the literature}

Rutledge \& Winkler (1989) made estimates of attenuation from VSP data in the Upper Basalt Series in the Vøring Plateau area of the eastern Norwegian Sea. From 451 to $1111 \mathrm{~m}$ below the sea-floor in $1289 \mathrm{~m}$ of water, they found 105 basalt flows with about $10 \%$ of the section comprising volcaniclastic sediments. Their estimates of overall scattering attenuation of $2.7 \times 10^{-4} \mathrm{~dB} / \mathrm{m}$ (effective $Q=25$ ) could be accounted for by scattering loss modelled from the sonic and density logs recorded in the well, leaving an intrinsic attenuation of less than $0.6 \times 10^{-4}$ $\mathrm{dB} / \mathrm{m}(\mathrm{Q}>115)$. Their $\mathrm{Q}$ of 25 is somewhat less than the estimated effective Q of 35 from the Lopra-1/1A VSP.

Pujol \& Smithson (1991) reported values of effective Q around 48, estimated from VSP data in thick, Columbia Plateau basalt sequences containing some interbedded clay zones. They also reported that scattering was the dominant loss mechanism, since elastic modelling was able to account for all the observed loss. The intrinsic losses in 


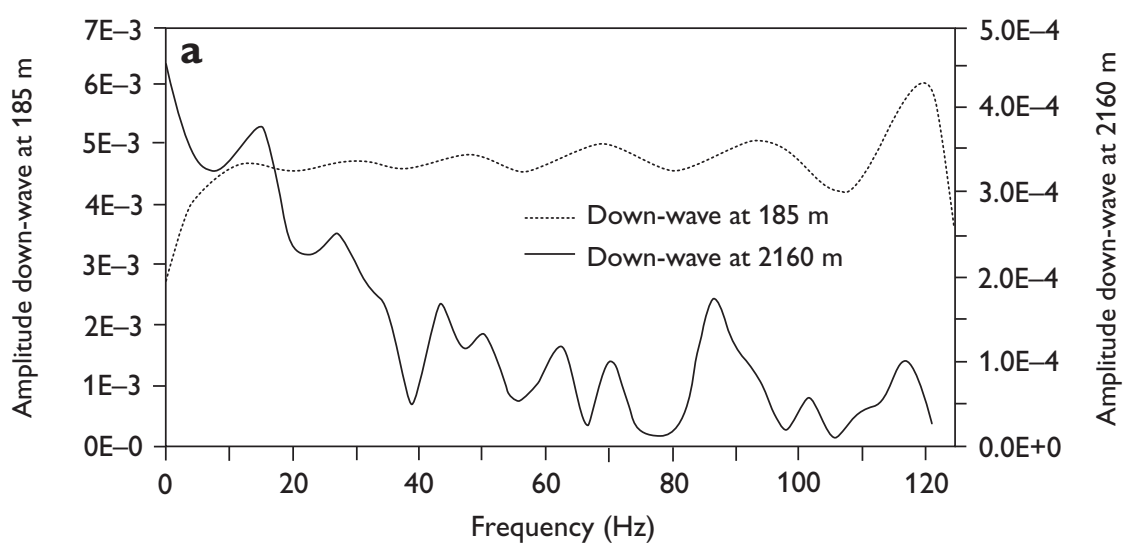

Fig. 13: a: Input and escaping wave spectra for a VSP downwave in a model similar to that in Fig. 12, but with a half space representing a sand unit at the base of the basalt sequence. The source wavelet is white over the full spectrum to Nyquist. The effects of scattering on the escaping wavelet are evident in the loss and the sidelobes. b: Regression of spectral ratios of the downgoing wave referenced to the input wavelet. The estimated effective $Q$ is 32 .

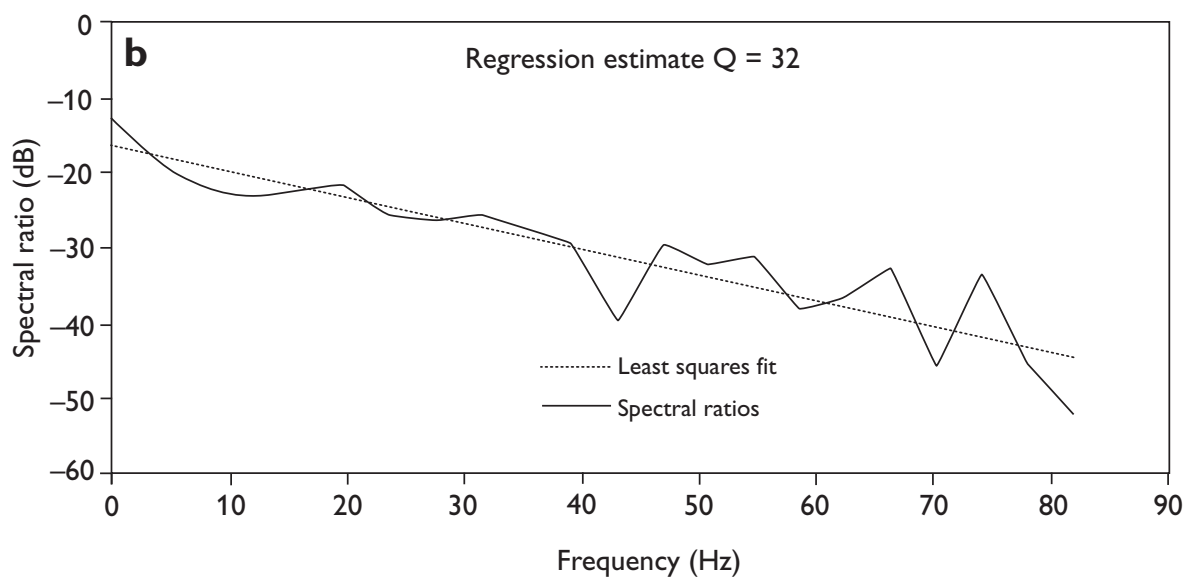

basalt were low and could not be determined from the field data.

The values of effective $Q$ estimated from Lopra-1/1A is bracketed by those reported in the literature, and the general trends and inferred loss mechanisms are consistent. It is almost certain that more data points from west of the Shetlands will result in a greater scatter of effective Q estimates, corresponding to the variety of basalt environments.

\section{Modelled offset VSP}

Figure 14 shows the wide-band $\mathrm{P}$ - and S-downwaves simulated at a horizontal array of receivers spaced $100 \mathrm{~m}$ apart and located $300 \mathrm{~m}$ below the base basalt in a half-space of sand. The volume injection source is located in a uniform, elastic layer, which is the overburden above the modelled basalt interval. The horizontal array of receivers in a $1 \mathrm{D}$ earth simulates a walkaway VSP shot into a single level geophone. Multiples are included in the simulation. The direct $P$ arrival is evident and its amplitude decays rapidly with offset due probably to a combination of strong geometrical spreading and critical angle effects at larger offsets in a medium with strong velocity contrasts.

Also evident is a strong, low-frequency event which dominates the shear record and is probably a mode conversion, propagating through the basalt sequence and emerging into the half space below. At the base basaltsand contact, it converts to a P-wave and is also recorded as a strong event on the $\mathrm{P}$-section, with an earlier arrival time. Both events are visible over the offset interval 2000 $4500 \mathrm{~m}$ but neither event can be traced to zero offset. The asymptotic velocity of about $3.4 \mathrm{~km} / \mathrm{s}$ is high and although it could correspond to the shear velocity of the shallow dolerites, which display the highest interval velocities in the sequence (Fig. 2), the event arrives before the direct shear arrival curve and it must therefore have a compressional leg for part of its ray path. The limited offset interval makes interpretation difficult and such conversions may be sensitive to the particular velocity-depth function, but the observation offers some encouragement 
Fig. 14. Full elastic model of single level walkaway VSP with the geophone located left panel are $\mathrm{P}$-waves while the right panel are $\mathrm{S}$-waves. The ray-traced first $\mathrm{P}$ and pure $S$ arrivals are superimposed. A mixed mode conversion can be seen with significant but low frequency amplitude over a limited offset interval. $300 \mathrm{~m}$ below the basalt-sand contact. The

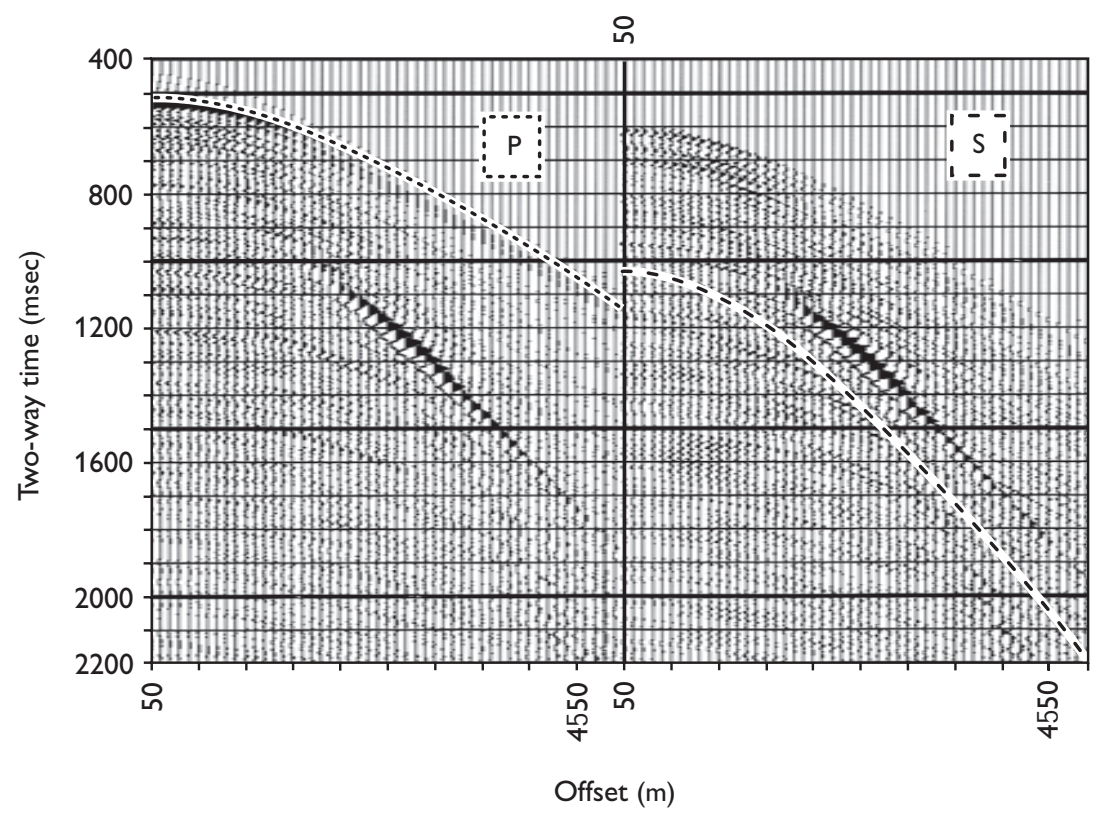

for the use of mode-converted shear waves to probebelow piled basalt flows, as described by Emsley et al. (1998).

\section{Conclusions}

The borehole seismic data recorded in the Lopra-1/1A well show that the average $\mathrm{P}$-wave velocity is high at 5248 $\mathrm{m} / \mathrm{s}$ from mean sea level to the deepest geophone level at $3510 \mathrm{~m} \mathrm{~KB}$. Amplitude loss over the stacked basalt flows is moderate, corresponding to an effective $\mathrm{Q}$ of 35 . However, this amplitude loss can be modelled by geometrical spreading and elastic scattering, implying that intrinsic attenuation is low. Persistent up-going events are evident within the interval logged by the VSP, even before wavefield separation, suggesting that lateral continuity of the basalt flow contacts is consistent over the several hundreds of metres that correspond to the VSP Fresnel zone radius. We observed an unambiguous tie between the VSP and a primaries synthetic seismogram which allows a detailed correlation between the stratigraphy revealed by the drillbit with the events on the VSP reflected wavefield. Both the pre-deepening targets at $920 \mathrm{~ms}$ and $1350 \mathrm{~ms}$ appear on the VSP at earlier times of $894 \mathrm{~ms}$ and $1330 \mathrm{~ms}$. Both events result from impedance contrasts within the volcanic sequence and it is likely that a strong reflection event, visible at $1412 \mathrm{~ms}$ TWT is also within the volcanic sequence. Its polarity indicates a decrease in acoustic impedance with depth so it is therefore unlikely to be basement. The prognosed depth of this event is $3732 \mathrm{~m} \mathrm{~KB}$, or $167 \mathrm{~m}$ below the final TD of the well at $3565 \mathrm{~m} \mathrm{~KB}$.
The unprocessed horizontal components suggest the presence of a persistent down-going shear wave, directly generated by the twin vibrators used as a VSP source, which in turn gives rise to up-going shear reflections. The $\mathrm{V}_{\mathrm{P}} / \mathrm{V}_{\mathrm{S}}$ ratio from the VSP is $1.8 \pm 0.1$ (estimated error), which is in good agreement with the estimate of $1.84 \pm 0.01$ (one standard deviation) obtained from the P-and S-sonic log data, and is rather constant over the logged interval. We estimate layer-induced anisotropy of about $5 \%$ due to the high elastic parameter contrasts in the basalt flows. Azimuthal anisotropy estimated from the dipole shear log is low, but the direction of the anisotropy is consistent with mapped master joint sets: the well-defined NE-SW direction gives way to the less well-defined NW-SE direction at about $540 \mathrm{~m} \mathrm{~KB}$. Our preferred explanation for the vertical $\mathrm{P}$-wavespeed being 10\% higher than the horizontal, as reported by Kiørboe \& Petersen (1995), is that vertical velocities are locally raised by the presence of fast, dolerite intrusions.

Reflection seismic data are difficult to process and indataset offers insight into the seismic properties of basalts which we anticipate will be of benefit in designing and processing reflection surveys, a topic which has attracted considerable interest but brings with it acknowledged challenges. An immediate result is that 'basalt', in seismic terms, cannot be represented by a uniform slab of hard rock a couple of kilometres thick. We observe challenges in the geometrical spreading, scattering losses, multiple development and spectral colouring which point towards low frequencies as the best hope for imaging beneath baterpret in basalt covered areas. The Lopra-1/1A borehole 
salts. This observation has been reported elsewhere in the literature but this paper may allow some more specific numbers to be applied to the basalt flows in the Faroese area.

On the positive side, intrinsic attenuation is low and propagation has been demonstrated through $3533 \mathrm{~m}$ of basalts, tuffs and volcaniclastic sequences. Coherent reflections have been tied from the VSP to the synthetic seismogram with confidence and another event below TD has been prognosed. Modelled offset VSP propagation also gave some hope for mode conversions, though with a limited offset range and low frequencies.

\section{Acknowledgements}

This work was carried out while the first author was engaged on a secondment with BP in Aberdeen. During this time he enjoyed considerable support from colleagues in BP, Shell and Schlumberger. In particular he would like to acknowledge many discussions and guidance from Matt Luheshi, Cameron Crook and Brian Mitchener. Chris Chapman answered many modelling questions and Fraser Louden recorded the VSP.

\section{References}

Backus, G.E. 1962: Long-wave elastic anisotropy produced by horizontal layering. Journal of Geophysical Research 67, 4427-4440.

Dansk Olie- og Gasproduktion \& Dansk Operatørselskab 1997: End of Well Report, Well Lopra-1, 80 pp. + 11 appendices. Thorshavn, Faroe Islands: Jardfeingi/Faroese Earth and Energy Directorate.

Emsley, D., Boswell, P. \& Davis, P. 1998: Sub-basalt imaging using long-offset reflection seismic data. 60th Meeting, European Association of Geoscientists and Engineers Expanded Abstract, 148.

Esmersoy, C., Koster, K., Williams, M., Boyd, A. \& Kane, M. 1994: Dipole shear anisotropy logging. 64th Annual International Meeting, Society of Exploration Geophysicists, Expanded Abstracts 1139-1142.

Folstad, P.G. \& Schoenberg, M. 1992: Low frequency propagation through fine-layering. 62nd Annual International Meeting, Society of Exploration Geophysicists, Expanded Abstracts 12781281 .
Folstad, P.G. \& Schoenberg, M. 1993: Scattering from a set of anisotropic layers to second order in frequency. 55th Meeting, European Association of Exploration Geophysicists, Extended Abstracts Paper, 105 only.

Kennett, B.L.N. 1974: Reflections, rays and reverberations. Bulletin of the Seismological Society of America 64, 1685-1696.

Kennett, B.L.N. 1983: Seismic wave propagation in stratified media, 342 pp. Cambridge: Cambridge University Press.

Kiørboe, L. \& Petersen, S.A. 1995: Seismic investigation of the Faroe basalts and their substratum. In: Scrutton, R.A. et al. (eds): The tectonics, sedimentation and palaeoceanography of the North Atlantic region. Geological Society Special Publication (London) 90, 111-123.

Larsen, L.M., Waagstein, R., Pedersen, A.K. \& Storey, M. 1999: Trans-Atlantic correlation of the Palaeogene volcanic successions in the Faeroe Islands and East Greenland. Journal of the Geological Society (London) 156, 1081-1095.

Newman, P. 1973: Divergence effects in a layered earth. Geophysics 38, 481-488.

Nielsen, P.H., Stefánsson, V. \& Tulinius, H. 1984: Geophysical logs from Lopra-1 and Vestmanna-1. In: Berthelsen, O., NoeNygaard, A. \& Rasmussen, J. (eds): The Deep Drilling Project 1980-1981 in the Faroe Islands, 115-135.Tórshavn: Føroya Fróðskaparfelag.

O’Doherty, R.F. \& Anstey, N.A. 1971: Reflections on amplitudes. Geophysical Prospecting 19, 430-458.

Planke, S. \& Cambray, H. 1998: Seismic properties of flood basalts from Hole 917A downhole data, southeast Greenland volcanic margin. In: Sanders, A.D., Larsen, H.C. \& Wise, S.W. Jr. (eds): Proceedings of the Ocean Drilling Program, Scientific Results 152, 453-462.

Pujol, J. \& Smithson, S.B. 1991: Seismic wave attenuation in volcanic rocks from VSP experiments. Geophysics 56, 1441-1455.

Rasmussen, J. \& Noe-Nygaard, A. 1970: Geology of the Faeroe Islands. Danmarks Geologiske Undersøgelse 1. Række 25, 142 pp.

Rasmussen, J. \& Noe-Nygaard, A. 1990: The origin of the Faeroe Islands in text, pictures and on maps, 64 pp., 6 maps at 1:50 000. Copenhagen: Geological Survey of Denmark (also text in Faeroese and Danish).

Rutledge, J.T. \& Winkler, H. 1989: Attenuation measurements from vertical seismic profile data: leg 104, site 642. In: Eldholm, O. et al. (eds): Proceedings of the Ocean Drilling Program, Scientific Results 104, 965-972.

Thomsen, L. 1986: Weak elastic anisotropy. Geophysics 51, 1954 1966.

White, R. \& McKenzie, D. 1989: Magmatism at rift zones: the generation of volcanic continental margins and flood basalts. Journal of Geophysical Research 94, 7685-7729. 